\title{
Mecanizado de Acero AISI1020, Utilizando Buriles con Recubrimiento Monocapa de Nitruro de Vanadio
}

\author{
John H. Navarro-Devia( ${ }^{(1)}$, Willian A. Aperador ${ }^{(1) *}$ y Arnoldo Delgado(2). \\ (1) Grupo de Investigación Volta, Facultad de Ingeniería, Universidad Militar Nueva Granada, Carrera 11 \\ No. 101-80, Bogotá-Colombia (e-mail: jhnavd@gmail.com; g.ing.materiales@gmail.com). \\ (2) Grupo de Investigación Energía Alternativa, Facultad de Ingeniería, Universidad Militar Nueva Granada, \\ Carrera 11 No. 101-80, Bogotá-Colombia.
}

* Autor a quien debe ser dirigida la correspondencia

Recibido May. 17, 2016; Aceptado Jul. 13, 2016; Versión final Ago. 3, 2016, Publicado Feb. 2017

\begin{abstract}
Resumen
Acero SAE-AISI 1020 fue mecanizado por arranque de viruta, utilizando buril de acero rápido ASSAB 17 como sistema de referencia, y buril con recubrimiento monocapa de nitruro de Vanadio (VN). La monocapa fue depositada mediante técnica de deposición física en fase vapor magnetrón sputtering; y se midió la temperatura generada en el acero durante el mecanizado. Mediante microscopia electrónica de barrido se caracterizó la resistencia al desgaste de las herramientas. Se evaluó también mediante rugosímetro y microscopio de fuerza atómica las superficies obtenidas en el acero mecanizado. Como resultado principal se encontró que en los buriles de acero rápido recubrimientos con monocapa de VN, se reduce en la herramienta el desgaste adhesivo y la transferencia de energía durante el proceso de corte. Por lo tanto, la aplicación del recubrimiento de $\mathrm{VN}$ en el corte por arranque de viruta de metales ferrosos presenta las ventajas de incrementar la vida útil de la herramienta, elevar la calidad de la pieza, y reducir los tiempos y costos del proceso de corte.
\end{abstract}

Palabras clave: desgaste; monocapa; mecanizado; herramienta de corte; nitruro de vanadio

\section{Machining on AISI 1020 using Monolayer Vanadium Nitride Coated Tool Bit}

\begin{abstract}
AISI-SAE 1020 steel was machined using high speed steel ASSAB 17 tool bit as reference and a tool bit coated with vanadium nitride (VN) monolayer. The coating process was carried out by the Magnetron Sputtering Physical Vapor Deposition technique. The experiment was based on temperature measures during the chip removal, with both burins, the coated and uncoated tool. The wear resistance of the tools was characterized by using Scanning Electron Microscopy and workpieces' surfaces were evaluated using Atomic Force Microscopy and Roughness tester. It was observed that the use of monolayer VN coatings on high speed steel reduces the adhesive wear of the tool, and the energy transfer during the cutting process. For this reason, the VN coating offers advantages in its application to chip removal process of ferrous metals by increasing the tool life, improving the quality of work pieces, and reducing cutting process time and cost.
\end{abstract}

Keywords: wear; monolayer; machining; cutting tool; vanadium nitride 


\section{INTRODUCCIÓN}

En el mecanizado de materiales metálicos, uno de los criterios más importantes relacionados con la calidad del proceso y de la pieza trabajada es el acabado superficial obtenido, y depende en alto grado de los parámetros del corte, las propiedades de la herramienta, las características del material a trabajar, y la interacción entre estos factores (Halila et al., 2014; Ryan et al., 2015; Divakar et al., 2015). Buriles, brocas, fresas y otras herramientas de corte, al ser elaboradas por materiales con buenas propiedades, son utilizadas en los procesos de fabricación mediante el mecanizado por arranque de viruta, al permitir realizar desbastes y maquinar los productos (Nayak et al., 2014 y Vogtel et al., 2014). En la industria mundial algunos procesos de mecanizado requieren altas velocidades y profundidad de corte elevadas, ocasionando desgaste adhesivo superficial de la herramienta por las temperaturas alcanzadas durante la fricción y el arranque de viruta (Palanikumar y Davim, 2007)., reduciendo su vida útil, un problema que decrementa la calidad de la pieza y capacidad de producción (Pawade et al., 2008), dando la necesidad de inspeccionar el desgaste de la herramienta, las fuerzas de corte generadas, y el acabado superficial de la pieza (Mandal et al., 2011; Xie et al., 2015; Römer et al., 2012 y Murzin et al., 2015). Una solución a este problema es mejorar las propiedades mecánicas de la herramienta, como la dureza, resistencia al desgaste (Bobzin et al., 2014), la resistencia a la corrosión y reducción del coeficiente de fricción, mediante la aplicación de recubrimientos duros (Capote Rodriguez et al., 2015); con la finalidad de aminorar la velocidad del desgaste adhesivo, ampliando el control dimensional y superficial de las piezas mecanizadas (Pawade et al., 2008; Kant y Sangwan, 2014). Los recubrimientos se han venido utilizando comercialmente para prolongar la vida útil de elementos industriales como las herramientas de corte y componentes de máquinas, manteniendo una gran acogida y adecuación de forma industrial (Davies et al., 2007); reduciendo costos derivados de las paradas de pérdida del afilado la herramienta, manteniendo la calidad de las piezas mecanizadas, además logran reducir el impacto ambiental (Åstrand et al., 2004; Klocke et al., 2006; Bouzakis et al., 2009 y Smith et al., 1997).

El uso del Nitruro de Vanadio (VN) como recubrimiento duro ha sido temática de investigación desde inicios del Siglo, por lo que se han realizado diversos estudios referente a su obtención mediante magnetrón sputtering, la influencia de los parámetros de deposición en las estructuras y en las propiedades del recubrimiento, y la caracterización de las películas generadas mediante las técnicas de difracción de rayos $X(D R X)$, análisis prospectivo elástico (EPT), nanoindentación, pruebas de desgaste, espectroscopia de impedancia electroquímica (EIS) y curvas de polarización Tafel, entre otras; registrando que la superficie recubierta presenta una adecuada deposición del VN, es homogénea sin imperfecciones y posee una rugosidad superficial menor en comparación a otros recubrimientos con nitruros, reduciendo la susceptibilidad del sustrato a la influencia del entorno y la generación de corrosión; presentando alta dureza, bajo coeficiente de fricción incluso al incrementarse la temperatura y elevada resistencia a la corrosión, evidenciando que el uso de VN es recomendado como recubrimiento para mejorar la resistencia al desgaste, características deseadas en herramientas de corte en procesos de mecanizado (Liao et al.,2004; Posso et al.,2009; Caicedo et al.,2011; Brayek et al.,2012; Escobar et al., 2013a; Escobar et al., 2013b; Escobar et al., 2014a). Debido a esto se ha implementado recientemente el VN en insertos de Carburo (WC) para identificar las mejoras de las propiedades en aplicaciones industriales como el mecanizado de piezas, evaluando el desgaste de flanco ocasionado, y evidenciando su disminución en la herramienta recubierta comparado a la herramienta sin recubrir, concluyendo que el uso de este recubrimiento en elementos de mecanizado puede mejorar notablemente su desempeño en la industria (Escobar et al., 2014b); pero a diferencia de esta trabajo no se evaluó la calidad de los productos generados ni la temperatura durante el mecanizado.

La finalidad de este estudio fue evaluar el desempeño de un buril de acero rápido recubierto por un sistema monocapa de VN, registrando la temperatura del acero, además mediante la caracterización del acabado superficial obtenido, y el desgaste adhesivo de las herramientas, por medio de las técnicas MFA y MEB. Estos resultados fueron comparados con los presentados por una herramienta del mismo material sin recubrimiento y que fue usado en iguales condiciones y con los mismos parámetros del material recubierto.

\section{MÉTODOS Y MATERIALES}

En esta sección se hace mención a la metodología utilizada en desarrollo experimental de esta investigación y consta de: Deposición del recubrimiento, Proceso de Corte, y Caracterización.

\section{Deposición del recubrimiento}

Empleando la técnica de Magnetrón Sputtering R.F, a blancos de Vanadio (V) de 4 pulgadas de diámetro y pureza del $99,9 \%$ se les aplico un voltaje bias r.f negativo de $70 \mathrm{~V}$ a una potencia de $400 \mathrm{~W}$ para obtener recubrimiento de nitruro de vanadio (VN) como película delgada monocapa, depositado sobre buriles de 
acero rápido ASSAB 17 de 3/8 X 3" utilizados como sustrato. Este procedimiento se realizó a una presión base al interior de la cámara de vacío de $2,3 \times 10^{-5} \mathrm{mbar}$, presión total de trabajo de $3.6 \times 10^{-3} \mathrm{mbar}$, a una temperatura del sustrato de $400^{\circ} \mathrm{C}$, con ambiente de gases de trabajo de mezcla de $\operatorname{Ar}(93 \%)$ y $\mathrm{N}_{2}(7 \%)$ durante el crecimiento de la película. Con estos parámetros se obtuvo un espesor del recubrimiento de 1,2 $\mu \mathrm{m}$, sobre los buriles.

\section{Proceso de Corte}

En un centro de control numérico (CNC) se cilindro por arranque de viruta, varillas de acero AISI 1020 de 30 centímetros en forma de probetas cilíndricas con un diámetro de media pulgada con longitud de corte de 10 centímetros en condiciones de ausencia de lubricación, haciendo uso de la herramienta comercial y de la herramienta con el recubrimiento monocapa de nitruro de vanadio; con los parámetros de corte definidos en la Tabla 1, constantes para todas las pruebas y para ambas herramientas implementadas (Mandal et al., 2011). Las varillas de acero AISI 1020, fueron seleccionadas del mismo lote de producción nacional, disminuyendo la variación de los resultados por variaciones en las condiciones iniciales del acero, con dureza entre $82-83 \mathrm{HRb}$, y composición química porcentual de Carbono 0,1810\%, Manganeso 0,6319\%, Fosforo 0,0046\%, Azufre 0,0055\%, Silicio 0,1249\%, Cromo 0,0356\%, Níquel 0,0276\%, Molibdeno 0,0082\%, Cobre $0,0675 \%$, Estaño 0,0086\% y Vanadio 0,0012\%.

Por su parte la herramienta comercial ASSAB17 utilizada como sistema de referencia, fabricada en Suecia, se caracteriza por presentar una excelente capacidad de corte, resistencia al desgaste, alta dureza y estabilidad térmica. Pertenece al conjunto de aceros rápidos de alto contenido de Cobalto, con dureza entre HRc 67 - 70. Consta de 1 capa de recubrimiento con alta dureza superficial, buena resistencia al desgaste, estabilidad química, resistencia al calor, la oxidación, coeficiente de fricción, alta tenacidad, buena resistencia al calor, baja conductividad térmica, manteniendo su dureza a altas temperaturas; incrementando la vida útil de la herramienta de 3 - 5 veces, y permite aumentar la velocidad de corte de $20 \%$ a 70 , reduciendo los costos de del $20 \%$ a $50 \%$.

Luego se realizó el pulido de las probetas AISI 1020 mecanizadas, este proceso constó de dos cortes distintos realizados secuencialmente, y los parámetros implementados también están indicados en la Tabla 1, para una distancia de corte recorrida por cada herramienta de 382,95 m en total. Durante el proceso de corte se realizó la medición de la temperatura en la superficie de la barra de acero, utilizando un sensor infrarrojo con lente de convergencia que permite medir sobre un área de $0.8 \mathrm{~mm}^{2}$ a $10 \mathrm{~mm}$ de distancia, conectado a un equipo de cómputo para almacenar la información detectada, este sistema fue diseñado y utilizado en el estudio preliminar realizado por los autores (Navarro-Devia et al., 2016).

Tabla 1: Parámetros del proceso de arranque de viruta.

\begin{tabular}{|l|l|l|l|}
\hline \multicolumn{1}{|c|}{ Parámetro } & \multicolumn{1}{c|}{ Corte Base } & \multicolumn{1}{c|}{ Primer Pulido } & \multicolumn{1}{c|}{ Segundo Pulido } \\
\hline Velocidad de rotación Husillo & $500 \mathrm{rpm}$ & $500 \mathrm{rpm}$ & $1000 \mathrm{rpm}$ \\
\hline Velocidad de avance de la herramienta & $0,25 \mathrm{~mm} / \mathrm{rev}$ & $0,08 \mathrm{~mm} / \mathrm{rev}$ & $0,01 \mathrm{~mm} / \mathrm{rev}$ \\
\hline Penetración Diametral & $2,0 \mathrm{~mm}$ & $0,25 \mathrm{~mm}$ & $0,10 \mathrm{~mm}$ \\
\hline Distancia del Mecanizado & $14,7 \mathrm{~m}$ & $41,53 \mathrm{~m}$ & $326,72 \mathrm{~m}$ \\
\hline
\end{tabular}

\section{Caracterización}

Empleando un microscopio electrónico de barrido (MEB), JeoINeoScope JCM 5000 de caracterizo el desgaste de los buriles, con y sin recubrimiento VN. En el grado de pulido para poder medir las superficies del acero AISI1020 mecanizado e identificar la irregularidad se utilizó un rugosímetro PCE-RT 1200, un dispositivo dotado de un palpador de diamante, que desplaza una longitud sobre el material detectando la rugosidad superficial del acabado generado con cada herramienta; para estos ensayos se realizó la medición de la rugosidad en varios puntos aleatorios en las zonas mecanizadas, con longitud de onda (cutoff) de 2,5mm y longitud de palpación de $5 \mathrm{~mm}$ constantes para todas las muestras, realizando 20 mediciones en cada probeta mecanizada en función del tipo de herramienta

La dureza de los recubrimientos se midió en un sistema de nanoindentación Hysitron TI 950 Tribolndenter. Se empleó un indentador Berkovich tipo diamante. El indentador opera como triángulos equiláteros y tiene un radio en la punta de 0,1 $\mu \mathrm{m}$. Las condiciones de aplicación de la carga fue de una carga máxima carga de $5 \mathrm{mN}$, una velocidad de aproximación: $1.8 \mu \mathrm{m} / \mathrm{min}$, una tasa de carga y descarga de $0.01 \mathrm{mN} / \mathrm{min}$, y se hicieron cinco mediciones sobre el recubrimiento. El método escogido para determinar la dureza fue el desarrollado por Oliver y Pharr (1992). Para la realización de este trabajo se empleó un equipo de rayos-X, X'pert Pro de Panalytical, de uso general con las siguientes características: tubo con ánodo de cobre de 
longitud de onda de $1,54060 \AA$; un goniómetro de resolución estándar que contiene la geometría $(\theta-\theta)$ para la realización de mediciones, en configuraciones simétricas y asimétricas, y de película delgada con tamaño de paso de $0,005^{\circ}$; un detector proporcional de rayos- $X$ y como portamuestras se emplearon un goniómetro de micro-difracción con microscopio de alineación, soporte de películas delgadas; las medidas de DRX se realizaron en configuración geométrica Bragg-Brentano.

\section{RESULTADOS Y DISCUSIÓN}

En esta sección se hace mención a los resultados obtenidos y su respectivo análisis, para la evaluación del desempeño de los buriles y consta de los resultados de: Caracterización del Recubrimiento, Temperatura del Proceso, Rugosidad y acabado superficial, Caracterización mediante MFA y Caracterización mediante MEB.

\section{Caracterización del recubrimiento}

Para determinar la dureza se utilizó el método propuesto en los ensayos de nanoindentación realizados, y dan lugar a lo que se denominan curvas características de carga frente a desplazamiento, P-h. Estas curvas de carga-profundidad de penetración consisten en dos partes, una de carga y otra de descarga; que contienen información sobre la deformación elástica y deformación plástica del material, que puede usarse para calcular diversas propiedades mecánicas de los materiales, tales como, dureza y módulo elástico. Una vez conocida la profundidad de contacto, se obtiene el área de contacto a profundidad máxima, y con ella se puede calcular la dureza y el módulo elástico del material. En recubrimientos monocapas de nitruro de vanadio se observa, en la Fig. 1, la curva de carga y descarga obtenidas.

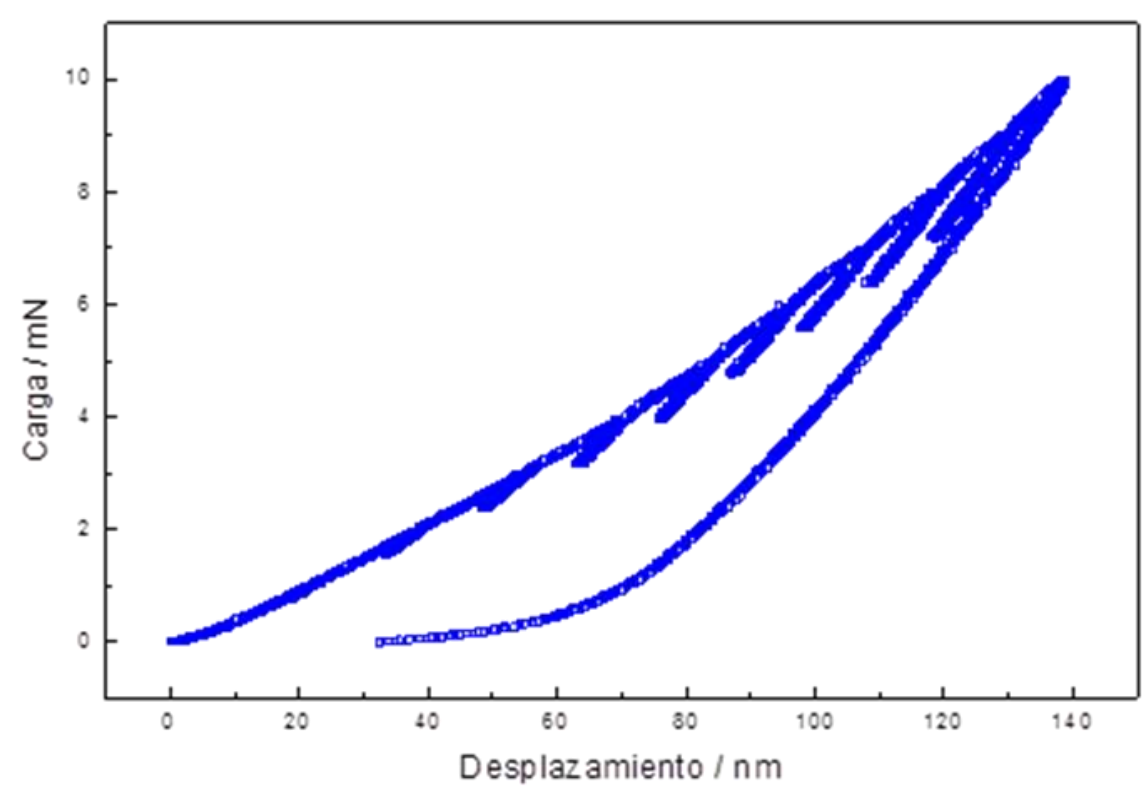

Fig. 1: Curvas de carga y descarga para los recubrimientos de monocapas de Nitruro de Vanadio, usada para la caracterización mecánica.

De la Fig. 1, mediante el método Oliver-Pharr (1992), se puede obtener el valor de dureza (H) que corresponde a $22 \mathrm{GPa}$ y módulo de elasticidad $(\mathrm{Er})$ con un valor de $209 \mathrm{GPa}$, un parámetro que se puede determinar a partir de los resultados es $\mathrm{H}^{3} / \mathrm{Er}^{2}=0.243$, el cual es proporcional a la resistencia de deformación plástica de los recubrimientos. Esta es una interesante característica cuando se trata de aplicaciones como el maquinado de piezas, presentando probabilidad de deformación plástica reducida debido a la alta dureza y bajo módulo de elasticidad, en este caso el valor de módulo de elasticidad es pequeño, y ya que cuando se aplica cierta carga sobre el material, ésta energía será distribuida sobre un área mayor. En la Fig. 2 se observa el espectro de difracción de rayos $\mathrm{X}$ de la película delgada, en donde se observa dos planos cristalinos (111) y (200) del nitruro de Vanadio. La orientación preferencial del plano (200) está relacionada con que las capas crecen de forma uniforme en aquellos planos donde la energía total sea mínima, esta energía es la sumatoria de las energías superficiales y de deformación, luego se establecen los planos (111) con menor valor de energía. El pico de mayor intensidad de difracción con orientación preferencial en (200) indica un alto grado de cristalinidad en esta orientación, y pertenecen a una estructura cubica del tipo FCC en la fase $\delta$-VN (Escobar et al., 2013a; Escobar et al., 2014c; Escobar et al., 2013c). 


\section{Temperatura del Proceso}

En la Fig. 3, se expone la temperatura de la operación de corte base; y los parámetros correspondientes al promedio de las pruebas realizadas, en la Tabla 2; identificando diferencias en las condiciones evaluadas, con el uso del buril convencional sin recubrir y el buril con recubrimiento de nitruro de Vanadio.

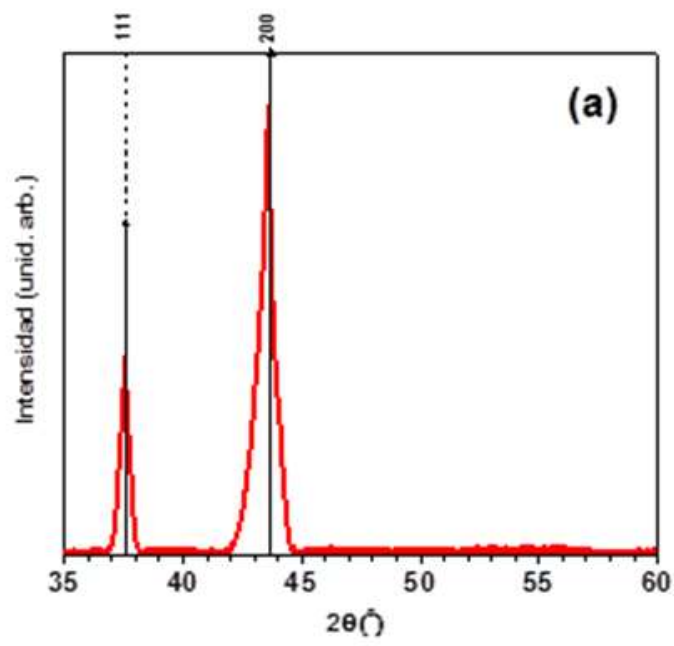

Fig. 2: Espectro de difracción de rayos $\mathrm{X}$ de la monocapa de nitruro de Vanadio depositada sobre el buril.

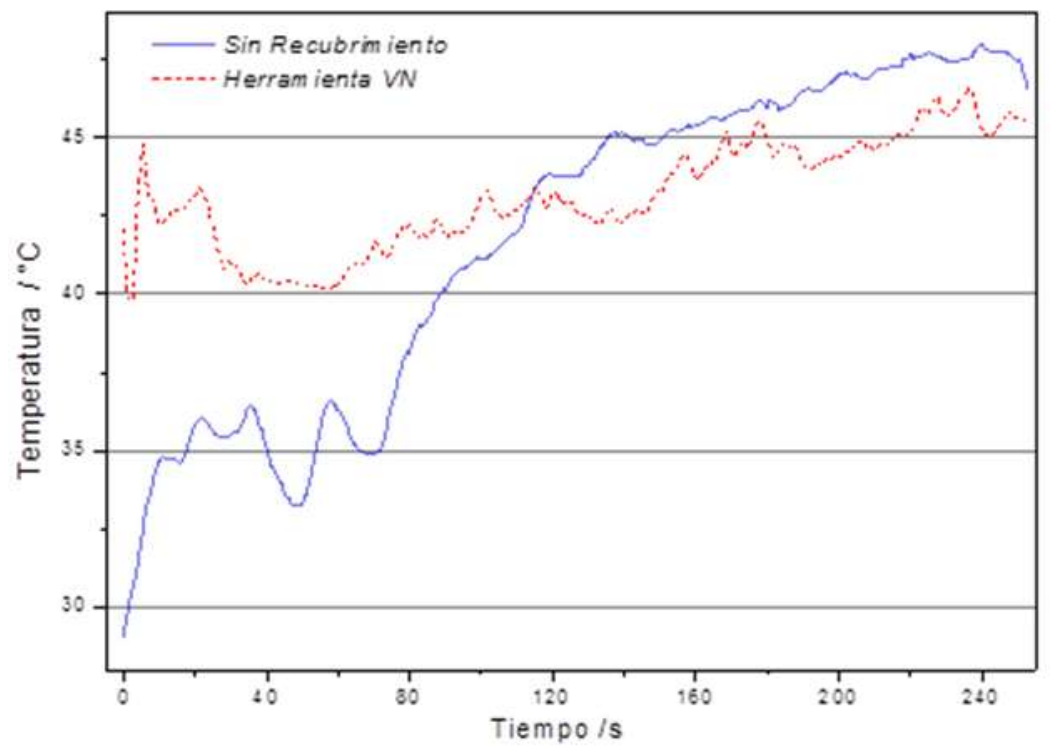

Fig. 3: Temperatura en el acero, durante el mecanizado utilizando buriles, con y sin recubrimiento de VN.

Tabla 2: Medición de la temperatura en el Buril durante el cilindrado de probetas de acero AISI1020.

\begin{tabular}{|l|l|l|}
\hline Parámetros & Buril sin recubrimiento & Buril recubierto con VN. \\
\hline Promedio $\left({ }^{\circ} \mathrm{C}\right)$ & 41,82 & 43,44 \\
\hline Cambios de temperatura $\left({ }^{\circ} \mathrm{C} / \mathrm{min}\right)$ & 3,85 & 0,427 \\
\hline Temperatura de Estabilización $\left({ }^{\circ} \mathrm{C}\right)$ & 48 & 45 \\
\hline Temperatura Máxima. $\left({ }^{\circ} \mathrm{C}\right)$ & 47,92 & 46,88 \\
\hline
\end{tabular}

La temperatura promedio del Acero, durante el proceso de corte, se incrementa al realizar el mecanizado con la herramienta recubierta, evidenciando que con el recubrimiento, energía del proceso es transmitida en mayor grado al metal mecanizado, pero la temperatura de estabilización se reduce de $48^{\circ} \mathrm{C}$ a $45^{\circ} \mathrm{C}$ debido a que el trabajo requerido sobre la pieza para desprender material se reduce, lo cual produce un mejor acabado superficial de la pieza, sin haber modificado los parámetros del corte. Adicionalmente, con la herramienta recubierta, se reduce la tasa de cambio de la temperatura, disminuyendo la deformación 
térmica y esfuerzos adicionales generados en la pieza mecanizada durante el corte, presentando mayor estabilidad del corte generado, aumento en la uniformidad del arranque de viruta, y ocasionando mejor acabado superficial de la pieza. El incremento de la temperatura máxima presentada al utilizarse el buril recubierto, revela que la herramienta recubierta puede generar mayor desprendimiento del material sin requerir variar el área de contacto con la pieza o las velocidades del corte. Incluso se presenta una mayor estabilidad de la transferencia de energía entre los elementos involucrados; y a menor tasa de cambio de temperatura, es mejor la calidad del proceso de corte y el acabado de la pieza (Davies et al. 2007).

\section{Rugosidad y acabado superficial}

Luego de realizar el corte y pulido del acero AISI 1020, con las distintas herramienta, se observó que con los buriles recubiertos, se generó viruta tipo continua, y reducción de la energía disipada a la viruta en la interfaz existente con la herramienta de corte, logrando un buen acabado superficial, con un bajo valor de rugosidad (Escobar et al., 2014a y Szymańska et al., 2014). En la tabla 3, se muestra que al implementar la herramienta recubierta con VN se presenta mejor acabado superficial, al reducir la irregularidad de la pieza trabajada hasta en $48 \%$; todo esto relacionado en el cambio de la temperatura del proceso en función del tipo de herramienta utilizada; la metodología desarrollada para evaluar la rugosidad de las superficies del acero AISI102 se indica en los detalles experimentales.

Tabla 3: Rugosidades en probetas de acero mecanizado con herramienta recubierta y sin recubrimiento de VN.

\begin{tabular}{|l|l|l|l|}
\hline Característica & $\begin{array}{l}\text { Rugosidad } \\
\text { Promedio }(\mu \mathrm{m})\end{array}$ & $\begin{array}{l}\text { Rugosidad } \\
\text { Máxima }(\mu \mathrm{m})\end{array}$ & $\begin{array}{l}\text { Rugosidad } \\
\text { mínima }(\mu \mathrm{m})\end{array}$ \\
\hline Sin recubrimiento & 7,61 & 18,3 & 2,46 \\
\hline Recubrimiento VN & 3,89 & 7,237 & 1,25 \\
\hline
\end{tabular}

\section{Caracterización mediante MFA}

Se realizó una caracterización comparativa del acabado generado por los dos tipos de herramientas utilizadas en el mecanizado del acero AISI 1020, mediante la técnica de Microscopia de fuerza atómica (MFA) en áreas de $1986 \mu \mathrm{m} 2$. En la Fig. 4. se observa el acabado superficial generado con la herramienta convencional, Fig. 3a.; y utilizando la herramienta con recubrimiento monocapa VN, Fig. 4b., una rugosidad superficial $R a$ de $1692 \mathrm{~nm}$ con la herramienta convencional, respecto a la rugosidad $R a$ de $577.64 \mathrm{~nm}$ generada con la herramienta recubierta; indico un mejor acabado superficial con lo que se interpreta un mejor comportamiento entre la herramienta y el material trabajado al disminuir la fricción del par tribológico (Quinto et al., 1987).

Demostrando la eficiencia del recubrimiento en la herramienta de corte, observando en la figura 4a una superficie irregular, con crestas pronunciadas y grandes defectos superficiales, mientras que en la figura $4 \mathrm{~b}$ se puede observar una superficie con mayor homogeneidad (Escobar et al., 2013d). Esto demuestra que se obtuvo una reducción de más del $50 \%$ de la rugosidad superficial al utilizar la herramienta recubierta.

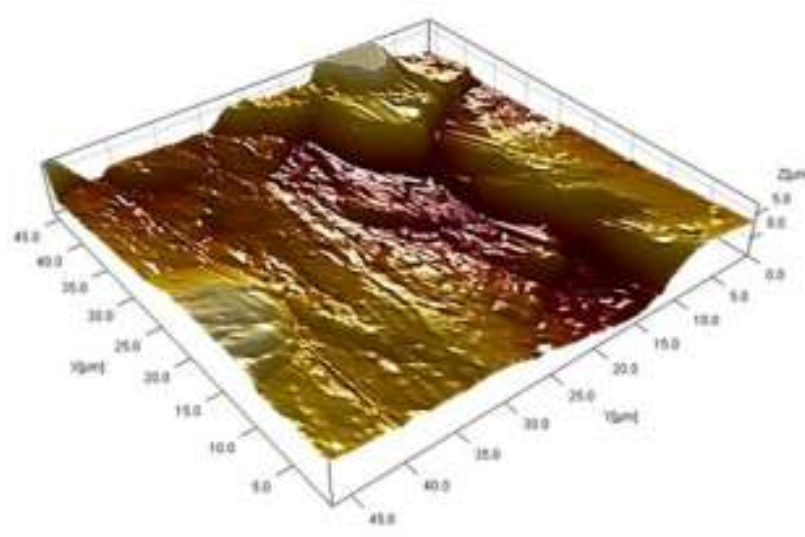

(a): $\mathrm{Ra}=1692 \mathrm{~nm}$

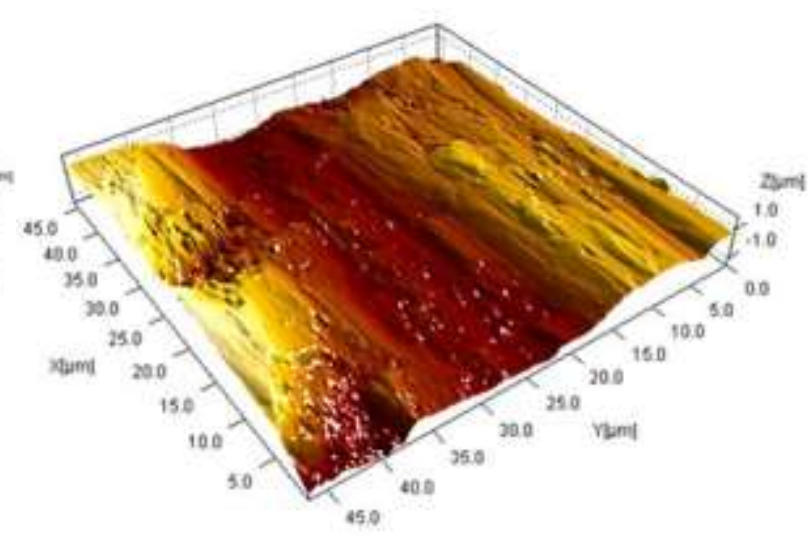

(b): $\quad R a=577.64 \mathrm{~nm}$

Fig. 4: Acabado superficial del acero AISI1020 visualizado en MFA, a) Pulido con buril convencional, b) Pulido con buril recubierta de VN. 


\section{Caracterización mediante MEB}

En la figura 5, a y b, se presenta la imagen morfológica obtenida luego del proceso de cilindrado y pulido, mediante microscopia electrónica de barrido (MEB), de los buriles utilizados en el corte. Al analizar comparativamente el estado de ambas herramientas, se manifiesta mayor desgaste y disminución del filo de la herramienta comercial, figura $5 \mathrm{a}$, presentando a $14,7 \mathrm{~m}$ de mecanizado un desgaste de flanco de $0,1 \mathrm{~mm}$ en el buril comercial y de $0,06 \mathrm{~mm}$ en el buril recubierto; evidenciado en el desprendimiento del material, generado en la superficie de incidencia de la herramienta y es ocasionado por frotamiento de la herramienta sobre la superficie mecanizada y a las temperaturas de trabajo (Álvarez, 2009). La mayor resistencia frente al desgaste luego del proceso de mecanizado se presentó en el buril recubierto con VN, figura $5 \mathrm{~b}$; esta característica del puede atribuirse a la formación de óxido de Vanadio, la cual actúa como lubricante entre las partes en contacto disminuyendo el coeficiente de fricción y a su vez el desgaste (Qiu, Li, et al., 2013), disminuyendo la probabilidad de presentar mecanismos de desgaste en la herramienta (Zhoua et al., 2010).

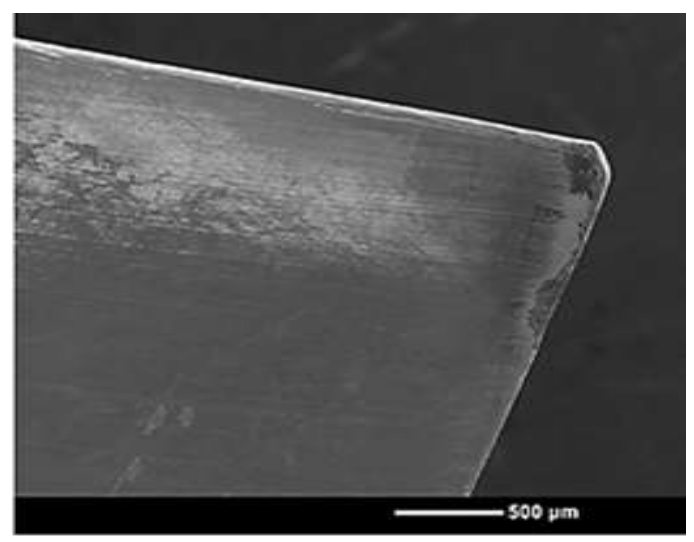

(a)

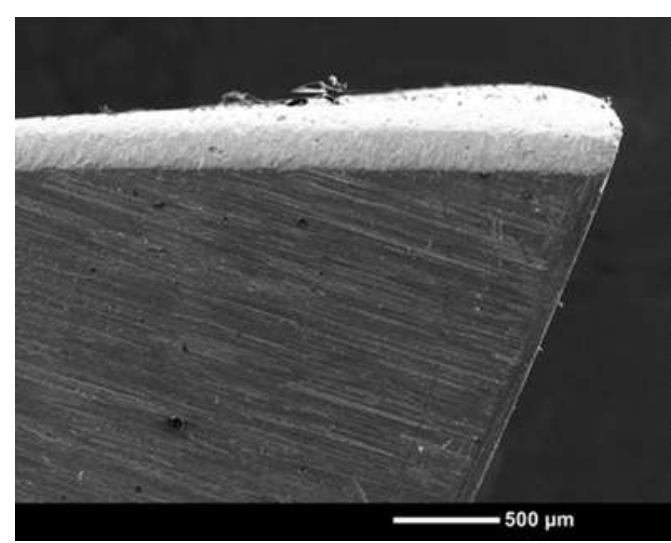

(b)

Fig. 5: Micrografías MEB luego del proceso de cilindrado de: a) Buril sin recubrir; b) Buril recubierto con VN.

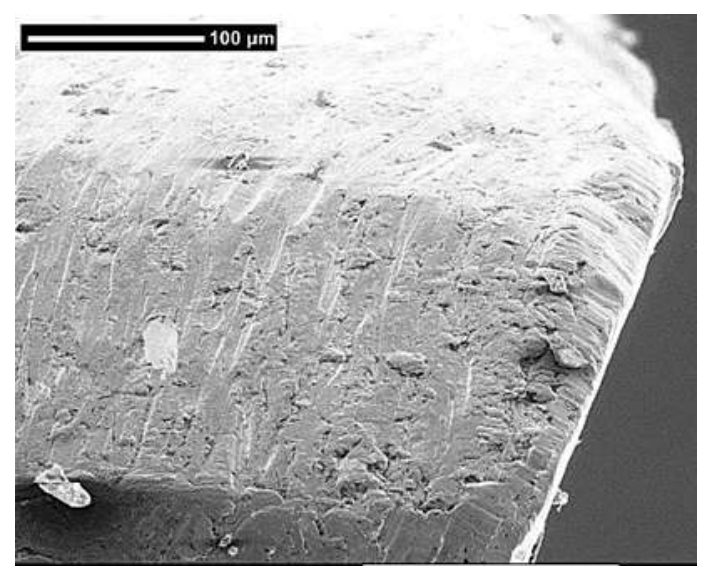

(a)

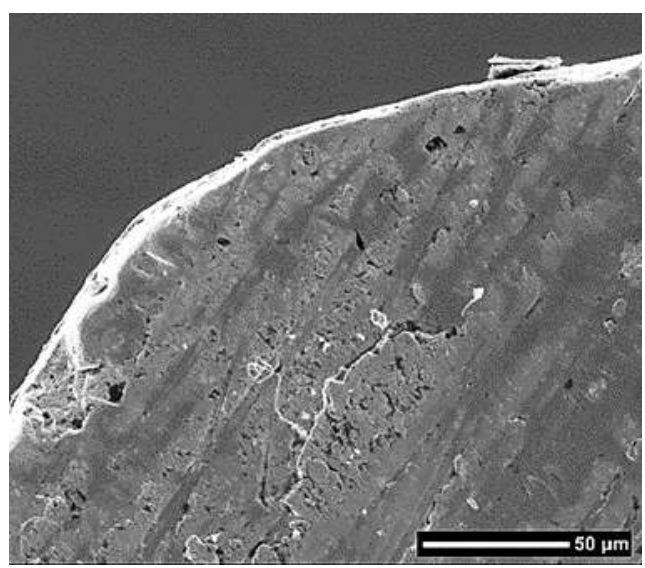

(b)

Fig. 6: Micrografías MEB del filo del Buril con recubierto VN utilizado: a) Vista 1; b) Vista 2.

En la Fig. $6 a$ y b, al realizar un mayor aumento en la punta analizada de la herramienta recubierta, se observan que los mecanismos de desgaste generados por el contacto durante el mecanizado del acero AISI 1020 , son el desgaste abrasivo, caracterizado por los surcos formados sobre la herramienta en la dirección de rotación de la pieza, y del desgaste adhesivo, evidenciado por la adhesión de partículas de la pieza trabajada sobre la superficie de la herramienta (Escobar et al., 2014b); aunque se observan puntos con desprendimiento de la capa de VN no se identifican zonas de deslaminación del recubrimiento ni exposición del sustrato, manteniendo una superficie homogénea, demostrando la resistencia al desgaste que otorga la monocapa de VN. Esta resistencia al desgaste del recubrimiento VN esta originada por su alta dureza y elevado módulo de elasticidad ocasionando reducción del coeficiente de fricción entre el buril y el acero mecanizado, lo que disminuye la temperatura del proceso con lo que se reduce el desgaste adhesivo, y aumentando la dureza superficial de la herramienta con lo que se reduce el desgaste abrasivo del proceso, obteniendo así una disminución de la zona de desgaste por las propiedades mecánicas del recubrimiento (Escobar et al., 2014b).

Por lo tanto, en el mecanizado de acero de bajo carbón AISI 1020 con herramienta recubierta monocapa de VN, se disminuyó en $48 \%$ la rugosidad de la pieza mecanizada con un aumento de la vida útil del buril del $40 \%$ al evaluar el desgaste de flanco. Comparando estos resultados respecto a los obtenidos del trabajo 
anterior realizado con monocapa de Nitruro de Hafnio (HfN) en las mismas condiciones de experimentación (Navarro-Devia et al., 2016)., se obtuvo que la herramienta con recubrimiento VN exhibe menores temperatura del acero mecanizado, flanco de desgaste, y rugosidad de la pieza mecanizada; evidenciando mejor desempeño frente al recubrimiento de $\mathrm{HfN}$, pero esto debe confirmarse realizando el estudio comparativo a diferentes parámetros de corte.

Todos los resultados mencionados evidencian, que con el uso del recubrimiento monocapa VN, al transmitir en mayor grado la energía generada en el corte al material metálico mecanizado, y reducir el desgaste sufrido por la herramienta, se eleva la calidad del proceso de corte y reduce las imperfecciones superficiales de la pieza mecanizada. Siendo la rugosidad de la pieza proporcional al crecimiento de la huella de desgaste, a la temperatura del acero, a la variación del radio del filo y el área de contacto del sistema material-herramienta-viruta; al aumentarse la resistencia al desgaste con el recubrimiento de VN se reduce el desgaste del buril ocasionando aumento de la vida útil y reducción de las imperfecciones superficiales del acero mecanizado.

\section{CONCLUSIONES}

La deposición de recubrimientos monocapa de $\mathrm{VN}$ en buriles de acero rápido reduce el desgaste e incrementa la vida útil de la herramienta por las propiedades mecánicas que posee; además perfecciona la calidad del producto manufacturado, acortando los tiempos y costos de producción; por lo cual su aplicación a nivel industrial ofrecería distintas ventajas competitivas y sustentables.

\section{AGRADECIMIENTOS}

Los autores agradecen a la Vicerrectoría de Investigaciones de la Universidad Militar Nueva Granada por la financiación de este trabajo, derivado del proyecto ING2100 vigencia 2016.

\section{REFERENCIAS}

Álvarez I. R., "Análisis por el método de elementos finitos de modelo simplificado de corte en aleaciones ligeras de interés Aeroespacial'. Tesis doctoral. Universidad nacional de educación a distancia, Departamento de Ingeniería de Construcción y Fabricación, España (2009)

Alves M.L., Ferreira B.B., Leta F.R., "Evaluación de Parámetros de Rugosidad usando Análisis de Imágenes de Diferentes Microscopios Ópticos y Electrónicos”, http://www.scielo.cl/pdf/infotec/v22n4/art14.pdf, ISNN: 0718-0764, Información Tecnológica, 22 (4), 129-149 (2011)

Åstrand M., Selinder T.I., Fietzke F., Klostermann H., "PVD-Al2O3-coated cemented carbide cutting tools", doi:10.1016/j.surfcoat.2004.08.021, Surface and Coatings Technology, Volumes 188-189, 186-192 (2004)

Brayek A., Tlilli B., Ghrib T., Nouveau C., "Investigation of vanadium and nitride alloys thin layers deposited by PVD", doi: 10.1051/epjconf/20122900042, EPJ Web of Conferences, 29 (42), 1-11 (2012)

Bobzin K., Bagcivan N., Ewering M., Brugnara R.H., Basturk S., "Comparison of (Ti,Al)N and (Ti,Al)N/YAl2O3 coatings regarding tribological behavior and machining performance", doi:10.1016/j.surfcoat.2014.08.070, Surface and Coatings Technology, 257, 58-62 (2014)

Bouzakis K.-D., Skordaris G. y otros 6 autores. "Ambient and elevated temperature properties of TiN, TiAIN and TiSiN PVD films and their impact on the cutting performance of coated carbide tools", doi:10.1016/j.surfcoat.2009.07.001,Surface and Coatings Technology, 204 (6-7), 1061-1065 (2009)

Caicedo J. C., Zambrano G., Aperador W., Escobar Alarcón L., y Camps E., "Mechanical and electrochemical characterization of vanadium nitride (VN) thin films", doi:10.1016/j.apsusc.2011.08.057, Applied Surface Science, 258 (1), 312-320 (2011)

Caicedo J. C., Zambrano G., Aperador W., Escobar Alarcón L., y Camps E., "Mechanical and electrochemical characterization of vanadium nitride (VN) thin films", doi:10.1016/j.apsusc.2011.08.057, Applied Surface Science, 258 (1), 312-320 (2011)

Capote Rodriguez G., Capote Mastrapa G., y Olaya J., "Resistencia al desgaste y a la corrosión de recubrimientos de DLC depositados sobre aceros AISI 304 y AISI 1020", http://www.rlmm.org/ojs/index.php/rlmm/article/view/573, ISSN: 2244-7113, Revista Latinoamericana de Metalurgia y Materiales, 35(1), 134-141 (2015) 
Davies M.A., Ueda T., M'Saoubi R., Mullany B., Cooke A.L., "On The Measurement of Temperature in Material Removal Processes", doi:10.1016/j.cirp.2007.10.009, CIRP Annals - Manufacturing Technology, 56 (2), 581-604 (2007)

Divakar Rao K., Udupa D.V y otros 4 autores. "Optical coherence tomography for shape and radius of curvature measurements of deeply curved machined metallic surfaces: a comparison with two-beam laser interferometry", doi:10.1016/j.optlaseng.2014.09.012, Optics and Lasers in Engineering, 66, 204-209 (2015)

Escobar C.A., Caicedo J.C., Aperador W., "Corrosion resistant surface for vanadium nitride and hafnium nitride layers as function of grain size", doi:10.1016/j.jpcs.2013.07.024,Journal of Physics and Chemistry of Solids, 75 (1), 23-30 (2014)

Escobar C.A., Caicedo J.C., Aperador W., Delgado A., y Prieto P., "Improve on Corrosion Resistant Surface for AISI 4140 Steel Coated with VN and HfN Single Layer Films", http://www.electrochemsci.org/papers/vol8/80607591.pdf, ISSN:1452-3981, International Journal of Electrochemical Science, 8 (6), 7591-7607 (2013)

Escobar C., Villareal M., Caicedo J.C., Aperador W., y Prieto P., "Novel performance in physical and corrosion resistance HfN/VN coating system", doi:10.1016/j.surfcoat.2013.02.002, Surface and Coatings Technology, 221, 182-190 (2013)

Escobar C., Villareal M., Caicedo J.C., Aperador W., y Prieto P., "Tribological and wear behavior of HfN/VN nanomultilayer coated cutting tools", doi:10.15446/ing.investig.v34n1.41101, Ingeniería e Investigación, 34 (1), 22-28 (2014)

Escobar C., Villareal M., Caicedo J.C., Esteve J., y Prieto P., "Mechanical and Tribological Behavior of VN and HfN Films Deposited via Reactive Magnetron Sputtering", http://econpapers.repec.org/article/wsisrlxxx/v_3a20_3ay_3a2013_3ai_3a03_3ap_3a1350040-1-1350040-

12.htm, ISSN: 0218-625X, Surface Review and Letters, 20(3), 1-12 (2013)

Escobar C., Villarreal M. y otros 4 autores. "Diagnostic of corrosion-erosion evolution for [Hf-Nitrides/VNitrides]n structures”, doi:10.1016/j.tsf.2013.07.081,Thin Solid Films, 545, 194-199 (2013)

Escobar C., Villareal M., Caicedo J.C., Aperador W., y Prieto P., "Mechanical Properties of Steel Surfaces Coated with HfN/VN Superlattices", doi:10.1007/s11665-014-1194-2, Journal of Materials Engineering and Performance, 23(11), 3963-3974 (2014)

Halila F., Czarnota C., Nouari M., "A new abrasive wear law for the sticking and sliding contacts when machining metallic alloys", doi:10.1016/j.wear.2014.03.013, Wear, 315 (1-2), 125-135 (2014).

Kant G., Sangwan K. S., "Prediction and optimization of machining parameters for minimizing power consumption and surface roughness in machining", doi:10.1016/j.jclepro.2014.07.073, Journal of Cleaner Production, 83, 151-164 (2014)

Klocke F., Gerschwiler K., Fritsch R., Lung D., "PVD-coated tools and native ester - an advanced system for environmentally friendly machining", doi:10.1016/j.surfcoat.2006.08.089, Surface and Coatings Technology, 201 (7), 4389-4394 (2006)

Liao M. Y., Gotoh Y., Tsuji H., y Ishikawa J., "Crystallographic structure and composition of vanadium nitride films deposited by direct sputtering of a compound target", doi:10.1116/1.1631473, Journal of Vacuum Science \& Technology A: Vacuum, Surfaces, and Films, 22, 146-150 (2004)

Mandal N., Doloi B., Mondal B., "Development of flank wear prediction model of Zirconia Toughened Alumina (ZTA) cutting tool using response surface methodology", doi:10.1016/j.ijrmhm.2010.12.001, International Journal of Refractory Metals and Hard Materials, 29, 273-280 (2011)

Murzin S. P., Shakhmatov E. V., Igolkin A. A., Musaakhunova L. F., "A Study of Vibration Characteristics and Determination of the Conditions of Nanopores Formation in Metallic Materials During Laser Action", doi:10.1016/j.proeng.2015.06.034, Procedia Engineering, 106, 266-271 (2015) 
Navarro-Devia J.H., Aperador W., y Delgado A. "Evaluación del Desempeño de Buriles con Recubrimiento Monocapas de Nitruro de Hafnio en el Proceso de Mecanizado", doi:10.4067/s0718-07642016000100014, Información tecnológica, 27(1), 127-138 (2016)

Nayak Sh. K., Patro J. K., Dewangan S., Gangopadhyay S., "Multi-objective Optimization of Machining Parameters During Dry Turning of AISI 304 Austenitic Stainless Steel Using Grey Relational Analysis", doi:10.1016/j.mspro.2014.07.086, Procedia Materials Science, 6, 701-708 (2014)

Oliver W. C., y Pharr G. M., "An improved technique for determining hardness and elastic modulus using load and displacement sensing indentation experiments", doi:10.1557/JMR.1992.1564, Journal of Materials Research, 7(6), 1564-1583 (1992)

Palanikumar K., Davim J., "Mathematical model to predict tool wear on the machining of glass fibre reinforced plastic composites", doi:10.1016/j.matdes.2006.06.018, Materials \& Design, 28 (7), $2008-2014$ (2007).

Pawade R.S., Joshi Suhas S., Brahmankar P.K., "Effect of machining parameters and cutting edge geometry on surface integrity of high-speed turned Inconel 718", doi:10.1016/j.ijmachtools.2007.08.004, International Journal of Machine Tools and Manufacture, 48 (1), 15-28 (2008)

Posso A., Yate L. y otros 6 autores. "Caracterización de Películas Delgadas de Nitruro de Vanadio (VN) Depositadas por Magnetrón Sputtering D.C”, http://revcolfis.org/publicaciones/vol41_1/4101017.pdf, ISNN: 0120-2650, Revista Colombiana de Física, 41 (1), 17-19 (2009)

Qiu Y., Zhang S., Li B., Wang Y., Lee Y., Li F., Zhao D. "Improvement of tribological performance of CrN coating via multilayering with VN”. doi:10.1016/j.surfcoat.2012.03.010, Surface \& Coatings Technology, 231, 357-363 (2013)

Quinto D. T., Wolfe G. J., Jindal P. C., "High Temperature microhardness of hard coatings produced by physical and chemical vapor deposition", doi:10.1016/0040-6090(87)90166-0, Thin Solid Films, 153, 19-36 (1987)

Römer G.R.B.E., Arnaldo Del Cerro D. y otros 6 autores. "Picosecond Laser Machining of Metallic and Polymer Substrates for Fluidic Driven Self-Alignment”, doi:10.1016/j.phpro.2012.10.082, Physics Procedia, 39, 628-635 (2012)

Ryan S. M., Szyniszewski S., Ha S., Xiao R., Nguyen T. D., Sharp K. W., Weihs T. P., Guest J. K., Hemker K. J., "Damping behavior of 3D woven metallic lattice materials", doi:10.1016/j.scriptamat.2015.03.010, Scripta Materialia, 106, 1-4 (2015)

Sai Chaitanya Kishore D., Prahlada Rao K., Mahamani A., "Investigation of Cutting Force, Surface Roughness and Flank Wear in Turning of In-situ Al6061-TiC Metal Matrix Composite", doi:10.1016/j.mspro.2014.07.175, Procedia Materials Science, 6, 1040-1050 (2014)

Smith I.J., Gillibrand D., Brooks J.S., Münz W.-D., Harvey S., Goodwin R., "Dry cutting performance of HSS twist drills coated with improved TiAIN", doi:10.1016/S0257-8972(96)03113-1, Surface and Coatings Technology, 90(1-2), 164-171 (1997)

Szymańska M., Gierałtowska S. y otros 4 autores. "Effect of reactive magnetron sputtering parameters on structural and electrical properties of hafnium oxide thin films", doi:10.1016/j.apsusc.2014.01.155, Applied Surface Science, 301, 28-33 (2014)

Vogtel P., Klocke F., Lung D., "High Performance Machining of Profiled Slots in Nickel-Based-Superalloys", doi:10.1016/j.procir.2014.03.061, Procedia CIRP, 14, 54-59 (2014)

Xie L. J., Ren X., Shen M. X., Tu, L. Q. "Parameter correlation of high-temperature creep constitutive equation for RPV metallic materials”, doi:10.1016/j.jnucmat.2015.05.032, Journal of Nuclear Materials, 465, 196-203 (2015)

Zhoua Z., Rainfortha W.M. y otros 4 autores. "Wear and friction of TiAIN/VN coatings against Al2O3 in air at room and elevated temperatures”, doi:10.1016/j.actamat.2010.01.020, Acta Materialia, 58, 2912-2925 (2010) 